\author{
M. Grosse ${ }^{\mathrm{a}}$, B. Schillinger ${ }^{\mathrm{b}}$, P. Trtik ${ }^{\mathrm{c}}$, N. Kardjilov ${ }^{\mathrm{d}}$, M. Steinbrück ${ }^{\mathrm{a}}$ \\ ${ }^{a}$ Karlsruhe Institute of Technology, Institute for Applied Materials/Applied Material Physics, Eggenstein-Leopoldshafen, Germany \\ ${ }^{\mathrm{b}}$ Technical University Munich, Physics Department, Garching, Germany \\ ${ }^{\mathrm{c}}$ Paul Scherrer Institut, Neutron Imaging and Activation Group, Villigen, Switzerland \\ ${ }^{\mathrm{d}}$ Helmholtz Zentrum Berlin, Institute for Applied Materials, Berlin, Germany
}

\title{
Investigation of the 3D hydrogen distribution in zirconium alloys by means of neutron tomography
}

\author{
Paper presented at the Symposium "Tomographic and Radiographic Imaging with Synchrotron \\ X-rays and Neutrons" of the MSE 2018, 26-28 September 2018, Darmstadt, Germany
}

The fuel rod claddings in nuclear light water reactors are made of zirconium alloys. Corrosion of these alloys during operation and in particular high temperature oxidation during nuclear accidents results in the production of free hydrogen. The cladding can absorb this hydrogen. It affects the mechanical properties of the cladding material. Hydrogen embrittlement of these materials provides the risk of brittle fracture of the cladding by thermo-shock during emergency cooling. At KIT the behaviour of cladding materials under different hypothetical nuclear accident scenarios was investigated. One focus was on hydrogen absorption and distribution/re-distribution in the alloys. The hydrogen distribution was determined mainly by neutron tomography. Examples for the determination of the 3D hydrogen distribution in cladding tubes after loss of coolant accident simulation tests are given and discussed.

Keywords: Hydrogen; Zirconium; Neutron imaging; Loss of coolant accidents

\section{Introduction}

Hydrogen degrades the toughness of zirconium-based alloys. The reduction of ductility can become safety relevant for nuclear fuel claddings or pressure tubes made of zirconium based alloys during operation, accidents and long-term dry storage. Cladding materials absorb hydrogen by water corrosion during operation and by steam oxidation during accident scenarios. The reaction with water can be simplistically described by

$\mathrm{Zr}+2 \mathrm{H}_{2} \mathrm{O}=\mathrm{ZrO}_{2}+4 \mathrm{H}$

$4 \mathrm{H}=4 f \mathrm{H}_{\text {absorbed }}+2(1-f) \mathrm{H}_{2} \uparrow$

with $f$ being the uptake portion. Depending on temperature, absorbed hydrogen is precipitated as zirconium hydrides and, in particular in the high temperature $\beta$ phase, dissolved in the zirconium lattice. Figures 1 gives the $\mathrm{Zr}-\mathrm{H}$ phase diagram [1]. The related hydrogen concentrations in the $C_{\mathrm{H}}^{\text {metal }}$ metal for a certain hydrogen partial pressures are given in the diagram too. They were calculated applying Sieverts' law [2]:

$C_{\mathrm{H}}^{\text {metal }}=K_{\mathrm{s}}(T) \sqrt{p_{\mathrm{H}_{2}}}$

The Sieverts coefficient $K_{\mathrm{s}}(T)$ depends on temperature by an Arrhenius law [1]:

$K_{\mathrm{s}}(T)=\exp \left(\frac{\Delta S}{R}-\frac{\Delta H}{R T}\right)$

$\Delta S, \Delta H, R$ and $T$ are the solution entropy, the solution enthalpy, the molar gas constant and the temperature, respectively. $\Delta S$ and $\Delta H$ depends slightly on the cladding alloy and on the oxygen content in solid solution [1].

Several experimental methods provide information about hydrogen concentration and distribution in components made of zirconium alloys. The hydrogen concentration is often determined by hot extraction. The sample is heated up above the melting temperature. More or less all hydrogen is released after melting because the hydrogen solubility in the melt is many orders of magnitude lower than in the solid state. This destructive method delivers integral values of the hydrogen content. X-ray and neutron diffraction are applied to determine type, amount and distribution of zirconium hydrides in the cladding tubes. In [3] electron probe microanalysis, micro elastic recoil detection analysis and laser induced breakdown spectroscopy microprobe are applied too.

Several groups are applying neutron-imaging methods for the investigation of hydrogen in zirconium and its alloys [3-20]. The basis of this method is the large difference between the total microscopic neutron cross sections of hydrogen and zirconium. Neutron radiography and tomography are non-destructive. Minimal hydrogen concentrations of few wt.ppm and spatial resolutions of about $25 \mu \mathrm{m}$ can be reached in standard experiments. Currently, the methodical developments of neutron microscopes like at the POLDI facility at PSI Villigen initially dedicated to strain measurements [21] improve the spatial resolution to a level that single zirconium hydride precipitates (length 10 to $1000 \mu \mathrm{m}$, thickness less than $3 \mu \mathrm{m}$ ) can be made visible [18].

Int. J. Mater. Res. (formerly Z. Metallkd.) 111 (2020) 1 
Neutron tomography was applied in the post-test examinations of cladding tubes from tests simulating design-basis large-break loss of coolant accidents (LOCA). According the nuclear safety rules, the coolability of a damaged reactor core has to be ensured during and after such a design basis accident. The coolability is proved if the temperature does not exceed $1200{ }^{\circ} \mathrm{C}$ during the accident, the oxidation level ECR (equivalent cladding reacted) of the cladding material is below $17 \%$ [22, 23]. Experiments performed about 15 years ago had shown that additional to the existing safety criteria, hydrogen absorption occurring after the burst of the cladding rods has to be taken into account [24-27]. The phenomenon known as secondary hydriding results in an embrittlement of the cladding material additionally to the embrittlement caused by oxygen uptake and oxide layer formation. Therefore, additional safety criteria considering the effects of hydrogen are under development.

Figure 2 provides a scheme of a risky LOCA scenario: The large break results in a loss of coolant and a pressure decrease in the reactor vessel to nearly normal pressure. The loss of water as coolant results in a rapid increase of the temperature. Then, the temperature decreases with the pressure drop. After this very short phase, the cladding is strongly heated because of heat stored in the fuel pellets inside the claddings. The inner pressure resulting from gaseous fission products and filling gas causes ballooning at hot spots of the cladding tube. This effect becomes strong when the temperature exceeds $800^{\circ} \mathrm{C}$ at which point the $\alpha$ to $\beta$ phase transformation starts due to transformation induced plasticity. The ballooning proceeds until tube burst by plastic collapsing. An axial oriented macrocrack with a length of 10 to $20 \mathrm{~mm}$ and an opening of 1 to $2 \mathrm{~mm}$ is formed. Fission gases are released from the fuel rods through the burst opening. Steam penetrates into the burst crack and into the gap between fuel pellets and inner surface (gap width between 0.1 and several millimetres). The steam meets the fresh metallic inner tube surface, which also becomes oxidized by the steam. According to Eq. (1) hydrogen remains and is concentrated in the gap. A higher hydrogen partial pressure is connected with a higher hydrogen concentration in the remaining metallic zirconium as Eq. (3) describes. The increased hydrogen concentration in the cladding decreases the plasticity of the materials and reduces the thermo-shock stability of the cladding tube. The tube can crack brittlely caused by the thermo-stresses induced by the emergency cooling. This brittle fracture can lead to complete breaking of the cladding tube including cladding fragmentation. In this case, solid fission products would be released and complete or fragmented fuel pellets relocated. The coolability of the reactor core would no longer be possible if the accident proceeded in this way. Therefore, it has to be proofed that this fragmentation does not occur.

To achieve this proof, the QUENCH-LOCA subprogram of the QUENCH program [28] was initiated. In the QUENCH program at KIT, nuclear accidents are simulated experimentally on fuel rod simulator bundle scale. The typical bundle in the German KONVOI reactor geometry consists of 21 fuel rod simulators with lengths of $2.5 \mathrm{~m}$ arranged reactor typically.

QUENCH-LOCA bundles were tested under simulated loss of coolant nuclear accident conditions. Whereas the results of the first two tests (QUENCH-LOCA-0 and -01) using Zircaloy-4 claddings were already published in [19, 29], this paper focuses on the results of the QUENCH-LOCA-03 test using cladding tubes made of the opt. ZIRLO ${ }^{\circledR}$ alloy in the as received state. For this test, more extensive neutron tomography examinations of the tested cladding tubes were performed giving details of the hydrogen distribution not ob-

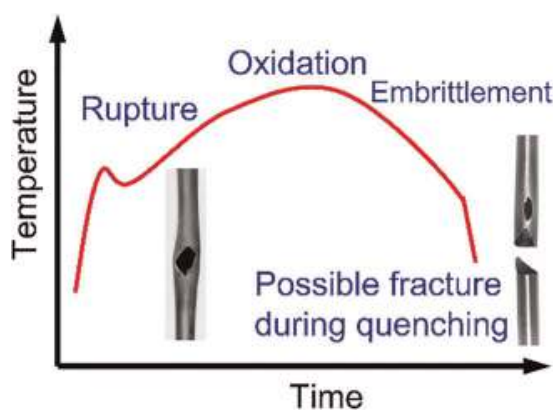

Fig. 2. Scheme of the scenario of a design-basis large-break loss of coolant accident.

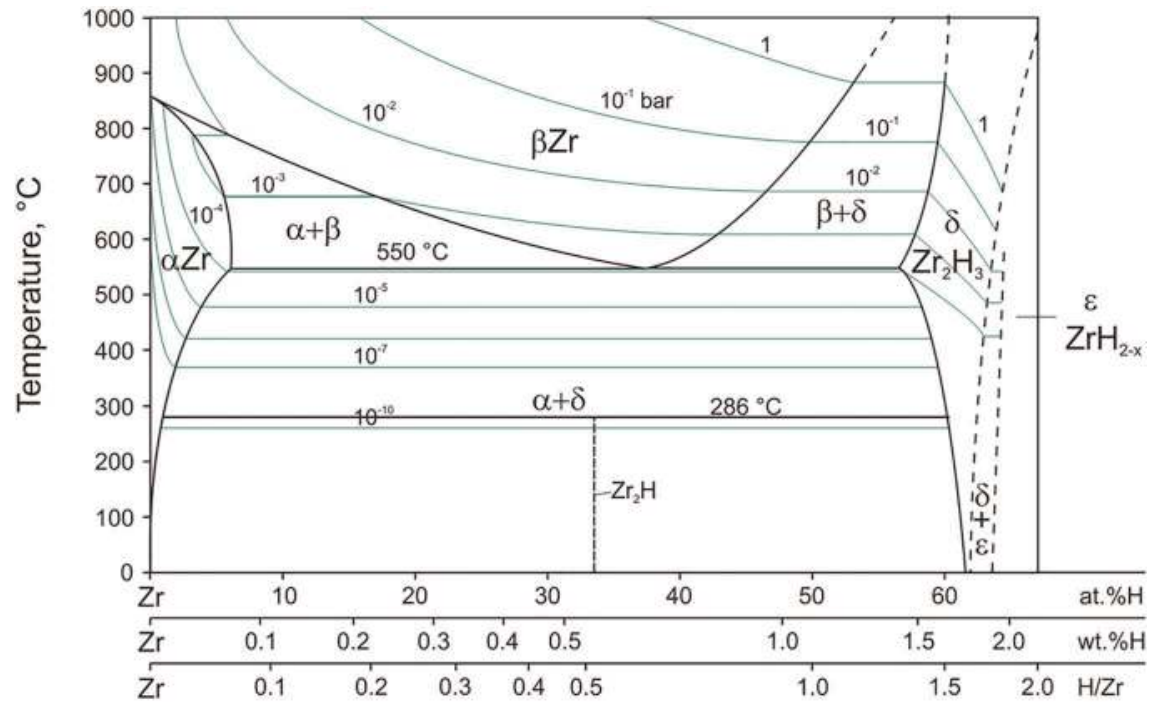

Fig. 1. $\mathrm{Zr}-\mathrm{H}$ phase diagram with equilibrium concentration for different environment hydrogen partial pressures isobars [1]. 
tained in the tomography measurements of cladding tubes of former QUENCH-LOCA tests. The QUENCH-LOCA-02 and -04 were performed with $\mathrm{M}^{\circledR}$ claddings and -05 with ZIRLO too. The QUENCH-LOCA-0 was quenched at the highest temperature. The tests QUENCH-LOCA-04 and -05 were performed with pre-hydrided cladding tubes (150 and 300 wtt.ppm, respectively). The test QUENCH-LOCA-05 was performed at slightly lower temperatures.

After an introduction into neutron imaging, results of the investigation of the 3D hydrogen distribution in these claddings obtained by means of neutron tomography are given.

\section{Contrast in neutron imaging}

The contrast in imaging is caused by differences of the attenuation of the initial beam. For neutron imaging differences in the total macroscopic total neutron cross sections and path length of the neutrons through the sample $\mathrm{s}$ at the different positions $x, y$ are used:

$I(x, y)=I_{0}(x, y) e^{-\Delta \Sigma_{\text {total }}(x, y) \mathrm{s}(x, y)}$

$I$ and $I_{0}$ are the intensities after and before passing the sample. The macroscopic total neutron cross-section is the sum over all isotopes $i$ of the microscopic total neutron cross-section $\sigma_{\text {total }}$ multiplied with the number density of the isotope:

$\Sigma_{\text {total }}=\Sigma_{i} N_{i} \sigma_{\text {total }, i}$

The total microscopic cross-section consists of the absorption cross-section $\sigma_{\mathrm{a}}$ and the cross-section of coherent and incoherent scattering $\sigma_{\mathrm{c}}$ and $\sigma_{\mathrm{i}}$, respectively:

$\sigma_{\text {total }}=\sigma_{\mathrm{a}}+\sigma_{\mathrm{c}}+\sigma_{\mathrm{i}}$

In contrast to X-rays where the absorption cross-section is orders of magnitudes larger than the scattering cross-section, all three cross-sections have to be taken into account for neutrons. Table 1 gives microscopic neutron cross-section values for natural isotope mixing for hydrogen, oxygen and zirconium at a wavelength of $0.1798 \mathrm{~nm}(E=25.3 \mathrm{meV})$ according to [30]. The coherent scattering strongly depends on the neutron wavelength. At critical wavelengths, the so called Bragg edge at which the scattering of a lattice plane $h k l$ is no longer possible $\left(\frac{\lambda}{2 d_{h k l}}>1\right), \sigma_{\mathrm{c}}$ falls immediately [31]. Even the correct values for the spectra applied at the different neutron sources are not available, the qualitative relation between the total cross-sections of the elements of interest given in Table 1 are comparable for cold neutrons. The contrast differences between hydrogen and zirconium is for cold neutrons even stronger than indicated at Table 1.

\section{Samples, experiments and data analysis}

Design-basis loss of coolant accidents (LOCA) with scenarios valid for the German KONVOI type nuclear reactors were simulated experimentally at fuel rod bundle scale in the framework of the QUENCH-LOCA program at KIT [19, 29]. The bundles consist of 21 vertically oriented fuel rod simulators with original cladding tubes. This paper focuses on the results of the QUENCH.L03 test performed with opt. ZIRLO ${ }^{\circledR}$ cladding tubes. ZIRLO $^{\circledR}$ is a Westinghouse zirconium alloy containing $0.7 \% \mathrm{Sn}$ and $1.0 \% \mathrm{Nb}$ as main alloying elements [32]. Figure 3 compares the temperature development measured at the hottest position of the inner and peripheral rods of the QUENCH-L03 test. The temperature gradient between inner and outer rods visible in Fig. 3 provides information about the temperature dependence of the material processes occuring during these simulation experiments. The temperature progression is similar to the QUENCH-L01, -L02, and -L04 tests allowing comparison between the behaviour of the different cladding materials.

The neutron imaging post-test investigations of the cladding tubes tested in the QUENCH-LOCA experiments were performed at the ANTARES facility at the FRM2 research reactor in Garching (Germany), the ICON and BOA facilities at the Swiss spallation neutron source SINQ, Villigen (Switzerland) and CONRAD facility at the BER research reactor, Berlin (Germany). Table 2 gives the spatial resolution and times per tomography of the measurements. The resolution depends on the neutron flux given by the neutron source, on the ratio between distance to the aperture slits and width of the aperture $L / d$, the detector available and the field of view (measurement of 2 or 3 rods at once). The neutron imaging setup

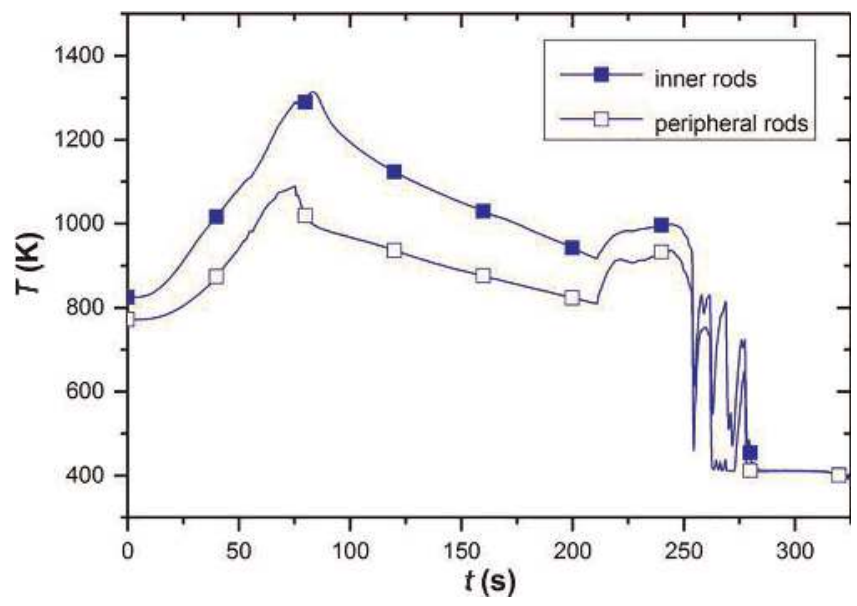

Fig. 3. Temperature development of the hottest position of inner and peripheral fuel rod simulators during the QUENCH-LOCA-03 test.

Table 1. Microscopic neutron cross-sections at a neutron wavelength of $0.1798 \mathrm{~nm}$ for hydrogen, oxygen and zirconium with natural isotope mixing.

\begin{tabular}{|c|c|c|c|c|}
\hline Element & $\sigma_{\mathrm{a}},\left(10^{-24} \mathrm{~m}^{2}\right)$ & $\sigma_{\mathrm{c}},\left(10^{-24} \mathrm{~m}^{2}\right)$ & $\sigma_{\mathrm{i}},\left(10^{-24} \mathrm{~m}^{2}\right)$ & $\sigma_{\text {total }},\left(10^{-24} \mathrm{~m}^{2}\right)$ \\
\hline $\mathrm{H}$ & 0.333 & 1.757 & 80.26 & 82.02 \\
$\mathrm{O}$ & 0.00019 & 4.232 & 0.0008 & 4.233 \\
$\mathrm{Zr}$ & 0.185 & 6.44 & 0.02 & 6.645 \\
\hline
\end{tabular}


Table 2. Spatial resolution and times per tomography measurements for the facilities used for the post-test examinations of the QUENCH-LOCA claddings.

\begin{tabular}{|c|c|c|c|}
\hline facility & source & spatial resolution & time needed \\
\hline ANTARES & FRM2, Garching, Germany & $\sim 50 \mu \mathrm{m}$ & 4 h per tomography \\
\hline CONRAD & $\begin{array}{c}\text { BER, } \\
\text { Berlin, Germany }\end{array}$ & $\sim 80 \mu \mathrm{m}$ & 14 per tomography \\
\hline ICON & $\begin{array}{c}\text { SINQ, } \\
\text { Villigen, } \\
\text { Switzerland }\end{array}$ & $\sim 80 \mu \mathrm{m}$ & 30 h per tomography \\
\cline { 1 - 4 } & & &
\end{tabular}

was chosen to have on the one hand the resolutions of the different measurements as comparable as possible and on the other hand to be good enough to be in the order of $1 / 10$ of the cladding tube wall thickness $(0.75 \mathrm{~mm})$ or slightly better.

The 3D reconstructions from the neutron radiography projections were performed using the muhrec software (ImagingScience). Because of the high neutron transmissions of the samples, beam-hardening effects can be neglected. Therefore, the CT number of the voxel can be taken as the total macroscopic neutron cross section were obtained. The dependence of $\Sigma_{\text {total }}$ on the hydrogen concentration was calibrated according to Eq. (6) as described in [8] for each facility. Calibration specimens were produced by annealing of cladding tube segments in very clean $\mathrm{Ar} / \mathrm{H}_{2}$ atmosphere at temperatures between 800 and $1200^{\circ} \mathrm{C}$. According Eq. (3), samples with different hydrogen concentrations were produced by varying temperature and hydrogen partial pressure. The hydrogen concentration of the samples were determined by measuring the weight gain. Six samples with number density ratios between hydrogen and zirconium $N_{\mathrm{H}} / N_{\mathrm{Zr}}$ in the range of 0.22 to 1.78 were produced according to Eq. (3). The linear dependence of $\Sigma_{\text {total }}$ on $N_{\mathrm{H}} / N_{\mathrm{Zr}}$ as can be derived from Eq. (6) was proved by the data. Using the correlations obtained by the calibration measurements, the hydrogen concentrations in the voxels can be calculated from the CT values.

Python-macros were written for the ImageJ software package for the statistical analysis of the data. A general problem is the relatively high standard deviation of the intensity values measured per pixel. Typical values are 200 to 1000 counts per pixel resulting in a standard deviation of a couple of percent. This data scattering is added to effect of the inhomogeneous hydrogen distribution on the transmission. Therefore, it was not sufficient to use the maximal voxel values for the discussion determined in a statistic analysis. In order to reduce the deviation caused by the counting statistics, the slice values averaged over the whole sample cross-section were used to determine maximal hydrogen concentrations in axial direction. In this way, the counting statistics play less of a role.

\section{Results}

Even though the paper is focused on the results of neutron tomography investigations on the hydrogen concentration and distribution in the claddings applied in the QUENCHLOCA-3 test, some further findings of this test and the posttest examinations have to be given. The burst of the rods occurred at temperatures of about $1117 \mathrm{~K}$. The scattering of the burst temperatures of the inner and outer rods were 13 and $39 \mathrm{~K}$, respectively. The outer oxide layers had thicknesses of 20 to $25 \mu \mathrm{m}$ on the inner and about $15 \mu \mathrm{m}$ on the outer rods as metallographic investigations performed after the neutron imaging experiments had shown. The oxide layer at the inner surface grows only close to the burst opening (10 to $20 \mathrm{~mm}$ from the edge of the burst) because the steam in the small gap between fuel pellets and inner cladding surface completely reacts immediately. The thickness of these inner oxide layers is only very small (less than $3 \mu \mathrm{m}$ ).

As an example, the axial distribution of the maximal and averaged values of the hydrogen concentrations are given in Fig. 4. The values are determined by a statistical analysis of the voxel values in the slices. The values determined are a folding from the hydrogen distribution and the counting statistic. Therefore, the maximal values are at positions with high hydrogen concentrations and maximal values in the Gaussian distribution of the counting statistics. Therefore, the mean value is much more robust against the counting statistics and gives reliable information.

Figures 5 compares views of the 3D reconstruction of the hydrogen distribution in the inner rods of the QUENCHL03 test. The positions with higher CT attenuation coefficient corresponding with a higher total macroscopic neutron cross-section caused by hydrogen enrichments are marked in blue. Two types of enrichments are visible.

- Bands of hydrogen enrichments of the first type extending over a large circumferential range can be found. These bands are oriented non-perpendicular to the tube axis. These enrichments start at positions where the inner oxide layer formed after burst ends. Some of them form almost closed rings (rod \#04 and \#06). The maximal circumferential averaged hydrogen concentrations found in these enrichments are about $450 \mathrm{wt}$.ppm.

- The second type of hydrogen enrichment extends in the axial direction only at one circumferential position, which can be seen in all of these projections.

In only one case of the peripheral rods investigated (\#10, $\# 13$, \#16 and \#19, given in Fig. 6) were hydrogen enrichments found, but with much lower concentrations (rod \#13). The points visible at rod \#16 are caused by remains of the pellet simulators at the inner surface, which were not removed completely before the tomography measurements.

\section{Discussion}

The results obtained for the QUENCH-L3 claddings agree qualitatively well with the hydrogen distribution found in the QUENCH-LOCA tests -L01 (Zircaloy-4 cladding), 

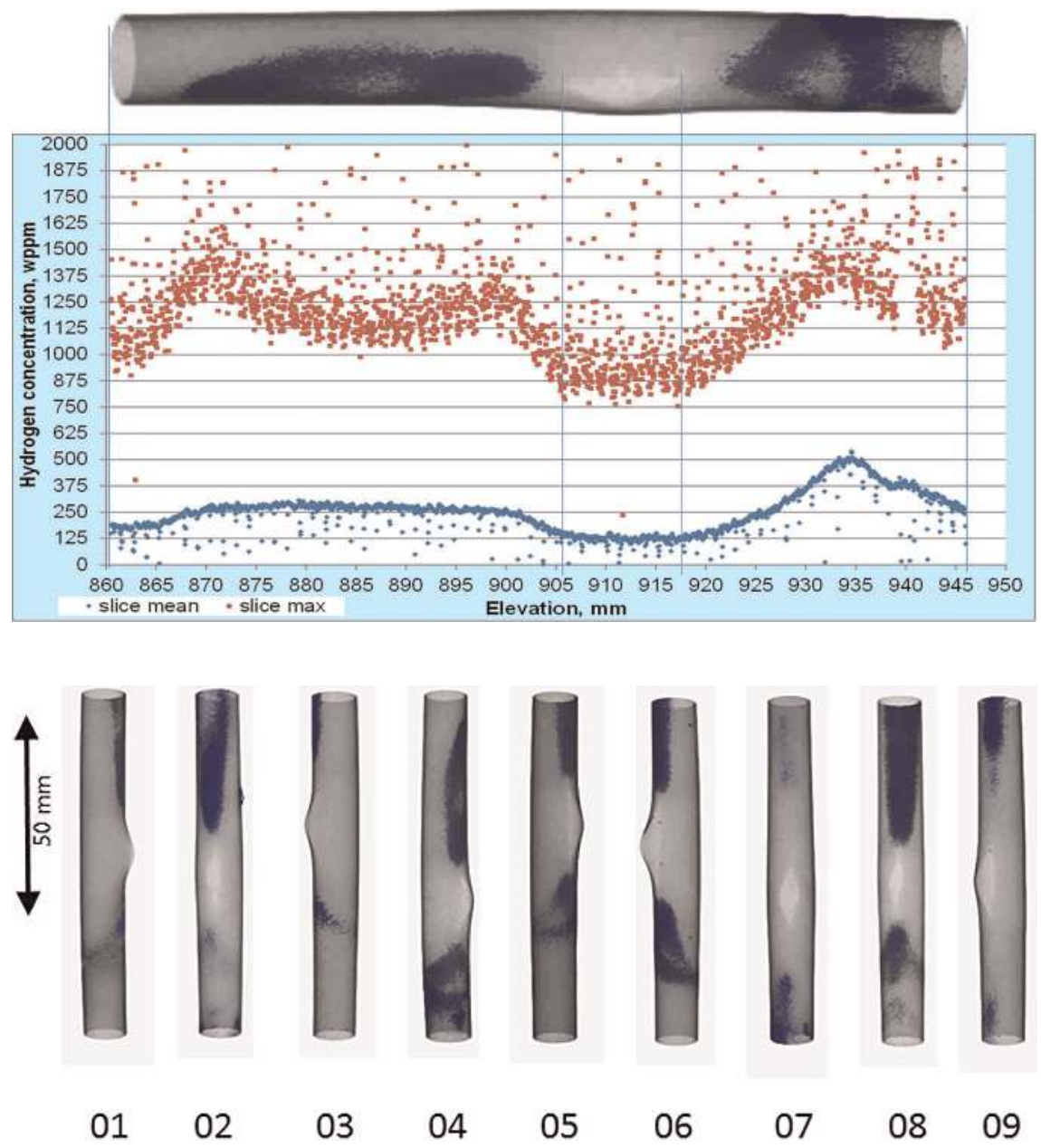

04

05

06

07

08
Fig. 4. Axial distribution of the maximal and averaged hydrogen concentration of the 3D reconstruction slices of sample QUENCHL04.

Fig. 5. 3D reconstructions of the hydrogen enrichments at the burst position of the claddings of inner fuel rod simulators tested in the QUENCH-LOCA-03 experiment.

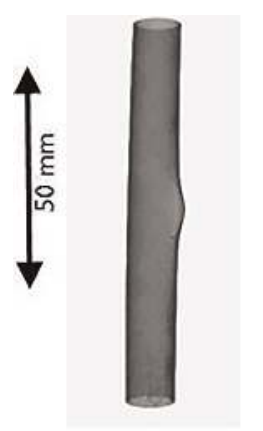

10

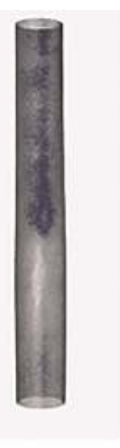

13

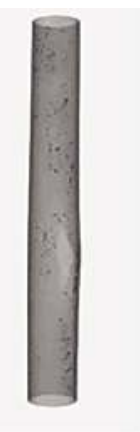

16

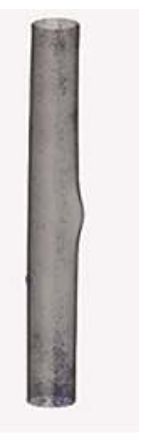

19

Fig. 6. 3D reconstructions of the hydrogen enrichments at the burst position of the claddings of peripheral fuel rod simulators tested in the QUENCH-LOCA-03 experiment.

L02 (M5 ${ }^{\circledR}$ cladding) and -L04 (pre-hydrided M5 ${ }^{\circledR}$ cladding) applying comparable temperature scenarios. All features were found in claddings of the other tests too. This indicates that the alloy as well as the initial hydrogen concentration have no strong influence on the secondary hydrogenation during LOCA.

The post-test metallographic investigations showed that the hydrogen enrichments are located at positions where the oxide layer on the inner surface around the burst openings of the cladding tubes fall below a critical thickness. Because of the small gap between pellet simulators and inner surface

(initially about $0.1 \mathrm{~mm}$ ) and the high temperatures (above $800^{\circ} \mathrm{C}$ ), the steam penetrating through the burst opening into this gap will be consumed rapidly. The oxide layer on the inner surface extends only several millimetres from the burst into the adjacent tube regions. The free hydrogen produced by the reaction with steam can be absorbed through the metallic surface into the cladding at positions where the oxide layers formed after the burst end. As own earlier experiments [10] have shown, even a sub-micrometre thick monoclinic oxide layer formed immediately at room temperature in air at a zirconium alloy hints a hydrogen uptake from the gas phase. In this experiments it was observed that the hydrogen uptake starts when the oxide layer disappears by diffusion of oxygen from the oxide layer into the remaining metallic zirconium alloy. These processes form the enrichments extended in circumferential directions.

The processes forming the hydrogen enrichments have been modelled. Details about the model are given in [33]. This type of enrichment is prototypical, i.e. they would be formed in a real Large Break-LOCA accident.

The other type of enrichments, the ones extended in the axial direction at only one circumferential position are caused by the unsymmetrical location of the pellet simulators made of zirconia. At the tube wall, positions touched by the pellets no steam but hydrogen can diffuse between pellets and inner surface. No oxide layers retarding hydrogen absorption are formed and the hydrogen can be taken up by the cladding. During real LB-LOCA, uranium oxide 
instead of zirconia pellets are in contact with the cladding tube wall. At accident temperatures, uranium oxide would react with metallic zirconium alloy reducing the oxygen content in the uranium oxide and forming a zirconium oxide layer. Under these conditions, hydrogen could not be absorbed. Therefore, the second type of hydrogen enrichments is not prototypical.

The finding of all seven QUENCH-LOCA tests that hydrogen absorption occurs more or less only in the inner cladding tubes agrees with the results of the other QUENCH-LOCA tests. It is obvious that the different temperature causes these differences. Generally, significant hydrogen enrichments are formed if the temperatures exceed about $1000^{\circ} \mathrm{C}$. The monoclinic to tetragonal phase transition in the zirconium oxide occurs in this temperature range. It can be concluded that only the formation of tetragonal zirconia results in strong hydrogen absorption. On the one hand, the reaction rate with steam is higher with tetragonal oxide layer than with monoclinic oxide layer. A higher hydrogen partial pressure in the gap between pellets and inner surface is the consequence and with it a higher hydrogen concentration in the metal, according to Sieverts' law. On the other hand, the diffusion of hydrogen in the tetragonal phase is higher than in the monoclinic one. Due to the short duration of an LB-LOCA, the higher diffusion rate in the tetragonal oxide results in a larger critical oxide layer thickness than in the monoclinic oxide phase, which still allows hydrogen absorption.

\section{Conclusions}

The hydrogen distribution in cladding tubes tested in the QUENCH-LOCA test series was investigated by means of neutron imaging. The following can be concluded from the results of the neutron tomography investigations of the cladding tubes tested in the QUENCH-L03 experiment:

- Two types of hydrogen enrichments were formed: Enrichment extended in the circumferential direction and enrichment at the burst side of the tube extended in the axial direction. Whereas the first type is prototypical for LB-LOCA scenarios valid for the German KONVOI type reactors, the second type would not occur in a real accident because in the simulation experiment no chemical interaction between cladding und pellets occurs.

- The maximal hydrogen concentrations in the prototypical enrichments with values of about 450 wt.ppm are not high enough to result in an unsuitable thermoshock resistivity.

- The comparison between the claddings of inner and of peripheral fuel rod simulators confirms the conclusions obtained from former QUENCH-LOCA tests that significant hydrogen enrichments are formed if the temperature exceeds a critical value of about $1000^{\circ} \mathrm{C}$. This critical temperature seems to be caused by the monoclinic to tetragonal phase transformation of the zirconium oxide occurring at this temperature.

The neutron imaging investigations were performed at the ANTARES facility at FRM-2 (TU Munich, Garching, Germany), the BOA and ICON facilities at SINQ (Paul Scherrer Institut Villigen, Switzerland) and at the CONRAD facility at BER (Helmholtz Centre Berlin, Germany). The authors thank for providing beam time and for the technical support during the neutron imaging experiments.

The German VGB (German acronym of "Verein der Grosskraftwerksbetreiber" - association of the large German utilities) sponsored the large-scale QUENCH-LOCA test series. The authors thank the QUENCH team at KIT for preparing and performing the tests as well as for the post-test examinations giving information about the cladding tube state after the tests additionally to the results described in this paper helping interpreting the results of neutron imaging.

\section{References}

[1] M. Steinbrueck: J. Nucl. Mater. 334 (2004) 58. DOI:10.1016/j.jnucmat.2004.05.007

[2] A. Sieverts, W. Krumbhaar: Chemische Berichte 43 (1910), 893. DOI: $10.1002 /$ cber. 191004301152

[3] J.C. Brachet D. Hamon M. Le Saux V. Vandenberghe, C. Toffolon-Masclet, E. Rouesne, S. Urvoy, J.-L., Bechade, C. Raepsaet, J.-L Lacour, G. Bayon, F. Ott: J. Nucl. Mater. 488 (2017) 267. DOI:10.1016/j.jnucmat.2017.03.009

[4] F. Gröschel, P. Schleuniger, A. Hermann, E. Lehmann: Nucl. Instrum. Methods A424 (1999) 215. DOI:10.1016/j.phpro.2013.03.035

[5] R. Yasuda, M. Matsubayashi, M. Nakata, K. Harada: J. Nucl. Mater. 302 (2002) 156. DOI:10.1109/TNS. 2005.844297

[6] E. Lehmann, P. Vontobel, A. Hermann: Nucl. Instrum. Methods A515 (2003) 745. DOI:10.1016/j.nima.2003.07.059

[7] E. Svab, G. Meszaros, Z. Somogyvari, M. Balasko, F. Körösi: Appl. Radiat. Isot. 61 (2004), 471. PMid:15246386; DOI:10.1016/j.apradiso.2004.03.072

[8] M. Grosse, E. Lehmann, P. Vontobel, M. Steinbrueck: Nucl. Instrum. Methods Phys. Res. Sect. A 566 (2006), 739. DOI:10.1016/j.nima.2006.06.038

[9] A. Agrawal, Y. Kashyap, P.S. Sarkar, A.N. Behra, M. Shukla, R.N. Singh, A. Sinha, J.K. Chakravartty: J. Nucl. Mater. 421 (2012) 47. DOI:10.1016/j.jnucmat.2011.10.047

[10] M. Grosse, S. Valance, J. Stuckert, M. Steinbrueck, M. Walter, A. Kaestner, S. Hartmann, J. Santisteban: MRS Online Proceeding Library Archive 1528 (2013). DOI:10.1557/opl.2013.364

[11] Z. Wang, U. Garbe, H. Li, R.P. Harrison, A. Kaestner, E. Lehmann: Metall. Mater. Trans. B 45 (2014) 532. DOI:10.1007/s11663-013-9866-0

[12] T. Smith, H. Bilheux, H. Ray, J.C. Bilheux, Y Yan: Phys. Proc. 69 (2015), 478. DOI:10.1016/j.phpro.2015.07.067

[13] C. Grünzweig, D. Mannes, A. Kaestner, F. Schmid, P. Vontobel, J. Hovind, S. Hartmann, S. Peetermans, E. Lehmann: Phys. Proc. 43 (2013) 231. DOI:10.1016/j.phpro.2013.03.027

[14] A.S. Tremsin, M. Morgano, T. Panzner, E. Lehmann, U. Filgers, J.V. Vallerga, J.B. McPhate, O.H.W. Siegmund, W.B. Feller: Nucl. Instrum. Methods A784 (2015) 486. DOI:10.1016/j.nima.2014.09.026

[15] S. Abolhassani, G. Bart, J. Bertsch, C. Degueldre, M. Grosse, L. Hallstadius, A. Hermann, G. Kuri, G. Ledergerber, C. Lemaignan, M. Martin, S. Portier, C. Proff, R. Restani, S. Valance, S. Valizadeh, H. Wiese: Zirconium in the Nuclear Industry: 17th Volume, Eds. B. Comstock and P. Barbéris, ASTM-STP1543 (2015) 540. DOI:10.1520/STP154320130007

[16] M. Grosse, M. Steinbrueck A. Kaestner: Nucl. Instrum. Methods A651 (2011) 315. DOI:10.1016/j.nima.2011.02.102

[17] M. Grosse, M. van den Berg, C. Goulet, A. Kaestner: J. Physics: Conference Series 340 (2012) 012106. DOI: $10.1088 / 1742-6596 / 340 / 1 / 012106$

[18] W. Gong P. Trtik, S. Valance, J. Bertsch: J. Nucl. Mater. 508 (2018) 459. DOI:10.1016/j.jnucmat.2018.05.079

[19] M. Grosse, J. Stuckert, M. Steinbrück, A. Kaestner: J. Nucl. Mater. 420 (2012) 575. DOI:10.1016/j.jnucmat.2011.11.045

[20] M. Grosse, J. Stuckert, C. Roessger, M. Steinbrueck, M. Walter, A. Kaestner: Zirconium in the Nuclear Industry: 17th Intern Symp., STP 1543, R. Comstock and P. Barberis, Eds. (2015) 1054. DOI:10.1520/STP154320120155

[21] U. Stuhr, M. Grosse, W. Wagner: Mater. Sci. Eng. A 437 (2006) 134. DOI:10.1016/j.msea.2006.04.069

[22] "Atomic Energy Commission Rule-Making Hearing, Opinion of the Commission", Docket RM-50-1, 28 December, (1973).

[23] „RSK-Leitlinien für Druckwasserreaktoren“, Ursprungsfassung (3. Ausgabe vom 14. Oktober 1981) mit Änderungen vom 15.11.1996 (in German)

[24] H.M. Chung: Nucl. Eng. Technol. 37 (2005), 327.

[25] F. Nagase, T. Fuketa: J. Nucl. Sci. Technol. 42 (2005), 209. DOI: $10.3327 /$ jnst.42.209 
[26] M. Billone, Y. Yan, T. Burtseva, R. Daum: "Cladding Embrittlement During Postulated Loss-of-Coolant Accidents," NUREG/ CR-6967 (2008). DOI:10.2172/946677

[27] C. Grandjean, G. Hache: "A State-of-the-Art Review of Past Programmes Devoted to Fuel Behaviour under Loss-of-Coolant Conditions. Part 3, IRSN report SEMCA 2008093, Saint Paul lez Durance Cedex, 2008.

[28] M. Steinbrück, M. Große, L. Sepold, J. Stuckert: Nucl. Eng. \& Des. 240 (2010) 1714. DOI:10.1016/j.nucengdes.2010.03.021

[29] J. Stuckert, M. Große, C. Rössger, M. Klimenkov, M. Steinbrück, M. Walter: Nucl. Eng. Des. 255 (2013) 185. https:// www.ncnr.nist.gov/resources/n-lengths. DOI:10.1016/j.nucengdes.2012.10.024

[30] J.R. Santisteban, L. Edwards, M.E. Fitzpatrick, A. Steuwer, P.J. Withers, M.R. Daymond, M.W. Johnson, N. Rhodes, E.M. Schooneveld: Nucl. Instrum. Methods Phys. Res. Sect. A 481 (2002), 765. DOI:10.1016/S0168-9002(01)01256-6

[31] J.P. Foster, R.J. Comstock, S.A. Worcester, G.P. Sabol: ZIRLO MATERIAL FOR LIGHT WATER REACTOR APPLICATIONS, United States Patent No 5,112,573 (1992).

[32] M. Grosse: Modelling of the hydrogen distribution in cladding tubes after LOCA, 19th International QUENCH Workshop, Karlsruhe, Germany, Nov. 19-21 (2013).

(Received November 5, 2018; accepted November 15, 2019)

\section{Correspondence address}

Mirco Grosse

IAM-AWP

Karlsruhe Institute of Technology

P.O. Box 3640

D-76021 Karlsruhe

Germany

Tel.: +4972160823884

Fax: +49721608 24567

E-mail: mirco.grosse@kit.edu

\section{Bibliography}

DOI 10.3139/146.111863

Int. J. Mater. Res. (formerly Z. Metallkd.)

111 (2020) 1; page 40-46

(C) Carl Hanser Verlag GmbH \& Co. KG

ISSN 1862-5282 